\title{
Bariatric surgery and the COVID-19 pandemic: SICOB recommendations on how to perform surgery during the outbreak and when to resume the activities in phase 2 of lockdown
}

\author{
Giuseppe Navarra ${ }^{1} \cdot$ Iman Komaei $^{1}$ (1) - Giuseppe Currò ${ }^{1} \cdot$ Luigi Angrisani $^{2} \cdot$ Rosario Bellini $^{3} \cdot$ Maria Rosaria Cerbone $^{4}$. \\ Nicola Di Lorenzo ${ }^{5}$ Maurizio De Luca ${ }^{6}$. Mirto Foletto ${ }^{7} \cdot$ Paolo Gentileschi $^{8} \cdot$ Mario Musella $^{9} \cdot$ Monica Nannipieri ${ }^{10}$. \\ Luigi Piazza ${ }^{11}$. Stefano Olmi ${ }^{12}$. Vincenzo Pilone ${ }^{13} \cdot$ Marco Raffaelli $^{14}$. Giuliano Sarro ${ }^{15}$. Antonio Vitiello ${ }^{16}$. \\ Marco Antonio Zappa ${ }^{17}$. Diego Foschi ${ }^{18}$
}

Received: 19 May 2020 / Accepted: 22 May 2020 / Published online: 8 June 2020

(C) Italian Society of Surgery (SIC) 2020

\begin{abstract}
The severe acute respiratory syndrome coronavirus 2 (SARS-CoV-2) and its related disease, coronavirus disease 2019 (COVID-19), has been rapidly spreading all over the world and is responsible for the current pandemic. The current pandemic has found the Italian national health system unprepared to provide an appropriate and prompt response, heavily affecting surgical activities. Based on the limited data available in the literature and personal experiences, the Società Italiana di Chirurgia dell'OBesità e Malattie Metaboliche (SICOB) provides recommendations regarding the triage of bariatric surgical procedures during the COVID-19 pandemic defining a dedicated path for surgery in morbidly obese patients with known or suspected COVID-19 who may require emergency operations. Finally, the current paper delineates a strategy to resume outpatient visits and elective bariatric surgery once the acute phase of the pandemic is over. Models developed during the COVID-19 crisis should be integrated into hospital practices for future use in similar scenarios. Surgeons are presented with a golden opportunity to embrace systemic change and to drive their professional future.
\end{abstract}

Keywords Coronavirus $\cdot$ SARS-CoV-2 $\cdot$ COVID-19 $\cdot$ Pandemic $\cdot$ Obesity $\cdot$ Bariatric surgery

Iman Komaei

komaei.iman@hotmail.com

1 Department of General Surgery, University Hospital of Messina “G. Martino”, Messina, Italy

2 Department of Public Health, University of Naples "Federico II", Naples, Italy

3 Bariatric Surgery Unit, Santa Chiara Hospital, Pisa, Italy

4 Department of Surgery, S. Maria Della Pietà Hospital, Casoria, Italy

5 Department of Surgical Sciences, University of Rome "Tor Vergata", Rome, Italy

6 Division of General Surgery, San Valentino Hospital, Montebelluna, Italy

7 Bariatric Unit, University of Padova, Padua, Italy

8 Obesity Unit, University of Rome "Tor Vergata", Rome, Italy

9 Advanced Biomedical Sciences Department, University of Naples "Federico II", Naples, Italy
10 Department of Clinical and Experimental Medicine, University of Pisa, Pisa, Italy

11 Department of General and Emergency Surgery, ARNAS "G. Garibaldi", Catania, Italy

12 Centre of Bariatric Surgery, San Marco Hospital, Zingonia, Italy

13 Department of Medicine, Surgery and Dentistry, University of Salerno, Salerno, Italy

14 Endocrine and Metabolic Surgery Unit, A., Gemelli University Hospital IRCCS, Rome, Italy

15 Magenta Hospital, Milan, Italy

16 Department of Surgery, University Hospital of Naples "Federico II", Naples, Italy

17 Clinical Epidemiology Unit, ISPRO, Florence, Italy

18 Department of Biomedical and Clinical Sciences, University of Milan, Milan, Italy 


\section{Introduction}

Coronaviruses $(\mathrm{CoV})$ are a vast family of non-segmented enveloped, positive-sense, single-stranded ribonucleic acid (RNA) viruses. Several coronavirus species have been known to cause diseases in humans that can range from mild respiratory diseases to more serious conditions such as severe acute respiratory syndrome (SARS) [1]. The new $\mathrm{CoV}$, severe acute respiratory syndrome coronavirus 2 (SARS-CoV-2), extracted from the lower respiratory tract samples of patients with pneumonia is a new entity that has not been previously identified in humans [2].

In December 2019, an epidemic of respiratory tract disease caused by SARS-CoV-2 was reported in Wuhan, China. Since then, the SARS-CoV-2-related disease, socalled coronavirus disease 2019 (COVID-19), has been rapidly spreading all over the world [3], and on March 11, 2020, the World Health Organization (WHO) declared the infection a pandemic. As of the 3rd of May, 2020, Italy has one of the highest rates of infection with more than 210,000 confirmed cases and nearly 29,000 deaths [4]. COVID-19 disease has been creating a rapid and distressing public health emergency in Italy due to the need for the assistance of an overwhelming number of infected patients and, at the same time, to secure treatment of all others non-deferrable medical conditions [5].

The pandemic has found the national health system unprepared to provide an appropriate and prompt response. A major priority during a pandemic is to reduce the medical and surgical procedures to the bare necessity. The need for dedicating economic, infrastructural, and medical resources to assist critically ill patients with COVID-19 has brought the need for reformulating the activities of many medical disciplines, even if not primarily involved in the management of the affected patient [6]. The limited availability of hospital beds, anesthesiologists, and ventilation devices in the COVID-19 pandemic era has also led to the suspension of all non-urgent/non-oncologic outpatient activities, the redistribution of the activities of the surgical wards, and limitation of surgical procedures only to the non-deferrable emergency and cancer operations $[7,8]$.

\section{COVID-19 and obesity}

It has been postulated that within the COVID-19 pandemic, obesity could be one of the pre-existing diseases associated with death and that the higher prevalence of obesity among the Italian population compared to China may account for the difference in mortality between the two countries [9]. In addition, obesity could shift severe
COVID-19 disease to younger ages [10]. Even if the direct consequences of obesity on the prognosis of COVID-19 patients has not yet been demonstrated, the H1N1 influenza experience that identified morbid obesity as a risk factor for a more aggressive course of the disease should alert surgeons on increased vigilance in taking care of these patients [11].

Obesity is not only a metabolic but also an attenuated inflammatory disease that can limit the ability to induce an appropriate response upon an immunologic challenge [12]. Moreover, it impacts lung mechanics, increasing the risk of respiratory complications. In fact, in COVID19-positive patients, obesity appeared to be a previously unrecognized risk for hospital and intensive care unit (ICU) admission in New York [13]. In a French study, the proportion of patients suffering from COVID-19 who required mechanical ventilation in ICU increased with BMI and was greatest in morbid obese population [14]. A greater anesthetic and surgical challenge, higher risks of postoperative complications, and possible long duration of hospital stay are only some of the numerous factors that have to be taken into account when planning surgical procedures in morbidly obese patients. The above-mentioned reasons highlight the importance of identification of a proper triage of surgical procedures for the treatment of morbidly obese patients during the COVID-19 pandemic.

\section{Study aim}

The purpose of the present document is to provide recommendations regarding the triage of surgical procedures in morbidly obese patients during the COVID-19 pandemic and define a dedicated path to safe surgery in patients with known or suspected COVID-19 who may require emergency surgery. Finally, we try to delineate a strategy for a safe resumption of bariatric surgery programs once the acute phase of the pandemic is over.

\section{Methods}

The present document is based on the opinion of surgeons that are currently members of the executive board of the Società Italiana di Chirurgia dell'OBesità e Malattie Metaboliche (SICOB). The given recommendations are based on the limited data available in the literature and on the personal experiences of the authors. The original draft was initially discussed by the authors and finally revised and approved by the first author. 


\section{Triage of surgical procedures in morbidly obese patients during COVID-19 pandemic}

Activities of bariatric surgical centers worldwide have been rearranged due to the COVID-19 pandemic. All non-urgent ambulatory activities have been suspended and weight-loss surgeries have been postponed [7, 8]. However, morbidly obese patients can still present with emergencies, either due to complications of previous weight-loss procedures or as a result of ex novo general surgical conditions.

American College of Surgeons [15] has proposed guidelines to help decision-making regarding the surgical procedures in various specialities and help to identify which procedures should be curtailed (Table 1). According to these guidelines, surgical procedures in the treatment of morbidly obese patients can be classified into:

\section{Emergency surgery}

- It must be reserved only for the critical cases when a delay in the surgery can be life-threatening or lead to end-organ damage. The indication of emergency surgery needs to be individualized and based on a highly accurate diagnosis.

2. Urgent surgery

- If possible, it should be delayed by a few days/weeks, particularly in COVID-19 infected patients until the infection clears.

- Alternative strategies, preferably under neuraxial or local anesthesia should be employed when possible (e.g., antibiotics, percutaneous drainage, endoscopic management, etc.).

- In non-infected morbidly obese patients requiring urgent surgery, several factors such as the necessity of postoperative ICU care, the need for transfusion of blood derivatives, and the presence of cardiocirculatory and respiratory comorbidities should be taken into account when planning the surgery.

3. Elective surgery

- These surgeries may be delayed for months without a threat to the patient's life or organ damage.

- Elective surgeries should not be performed at this time, especially in hospitals dedicated to the management of COVID-19 patients unless a clear separate pathway is dedicated to no-COVID-19 patients.

\section{Emergency surgical procedures during the COVID-19 pandemic}

All surgeons practicing in COVID-19 hospitals may be asked to perform an emergency procedure on patients with known or suspected infection. Therefore, operating room (OR) staff will have to comply with all the implemented pathways to decrease the risk of contagion and must be specifically trained to don, doff, and dispose of personal protection equipment (PPE).

The COVID-19 status of every patient should be considered before planning an operation and making decisions on the surgical approach. Upon presentation, patients should undergo an interview that will include history, physical examination, a patient questionnaire (directed at flu-like symptoms, body temperature in the past few days, travel, and exposure history), chest X-ray and/or chest CT and/or pulmonary ultrasound, as well as SPO2 determination [16]. All patients who need emergency surgery should undergo preoperative nasopharyngeal sample for SARS-CoV-2 and/ or a rapid test ( $\mathrm{IgG} / \mathrm{IgM}$ dosage) soon after admission.
Table 1 Triage of surgical procedures in morbidly obese patients in COVID-19 pandemic (American College of Surgeons' classification)

\begin{tabular}{lll}
\hline Surgical procedure & Specific bariatric conditions & General surgical conditions \\
\hline Emergency surgery & Perforated marginal ulcer & Hemorrhagic shock \\
& Bleeding & Septic shock \\
& Anastomotic or staple-line leak & Perforated viscus \\
& Intestinal obstruction (internal hernia) & Intestinal obstruction \\
& Gastric band perforation or prolapse & Risk of ischemic bowel \\
& Revisions for dysphagia & Necrotizing soft tissue infections \\
Urgent surgery & Severe GERD & Appendicitis \\
& Dehydration/malnutrition & Acute cholecystitis \\
& Slipped band & Cholangitis, choledocolithiasis \\
& Anastomotic strictures & Acute pancreatitis \\
& Sleeve gastrectomy & Diverticular disease \\
Elective surgery & primary roux en Y gastric bypass (RYGB) & \\
& One anastomosis gastric bypass-mini gastric & \\
& bypass (OAGB-MGB) & \\
& Biliopancreatic diversion & \\
& Gastric band & \\
& Revisions for weight gain & \\
\hline
\end{tabular}


If possible, any decision on the approach to follow should be deferred until the sample result is available $[17,18]$. In patients with negative tests, surgery can proceed as indicated in a non-dedicated OR with a laparoscopic approach. If surgery cannot be delayed until nasopharyngeal sample for SARS-CoV-2 is available, laparoscopic surgery is still an option, following the indicated guidelines and in the designated COVID-19 OR [19, 20]. In any case, the patient should be kept in theater until the result of the test becomes available to decide if the patient should be transferred to an ordinary ward or a dedicated COVID ward. The summarized path to safe surgery during the COVID-19 pandemic is illustrated in Fig. 1.
A dedicated OR must be identified and marked with clearly visible signs. We recommend a designated OR away from other theaters and an established direct circuit for the transport of infected patients to and back from the OR to keep them apart from non-infected ones. Negative pressure ORs would be ideal to minimize infection risk $[16,17]$. However, ORs are normally designed to have positive pressure air circulation. A high air exchange cycle rate ( $>25$ cycles/hour) contributes to effectively reduce the viral load within ORs [18]. Therefore, it is advised not to induce anesthesia in induction rooms since here air changes per hour are usually less.

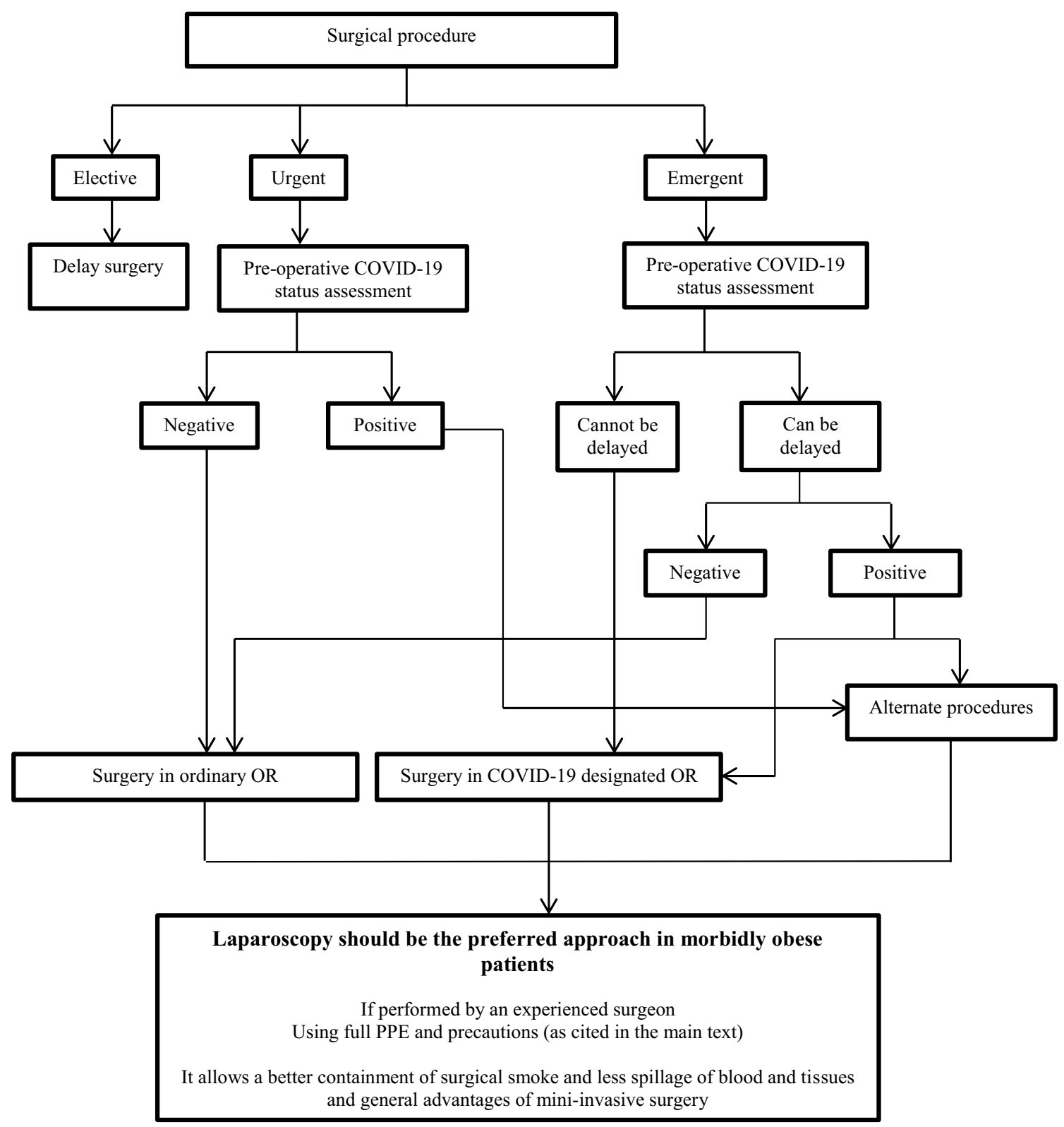

Fig. 1 The path to safe decision-making on the surgical approach in the acute phase of the COVID-19 pandemic 
All the required materials must be readily available in the OR. Disposable devices and materials are preferred. The operating team should minimize time spent within the OR itself, enter soon after the patient is intubated, should not leave until the operation is completed, and once out they should not re-enter. Movement of the staff between ORs is strongly contraindicated to avoid cross-infection and runners should be available outside the OR to supply extra materials needed by the surgical team while operating a patient with known or suspected COVID-19. Any indoor direct path utilized by the patient should be sanitized soon after. A dedicated specifically trained cleaning team is mandatory and should be ready $24 / 7$ [16-18].

In patients with positive formal test for COVID-19 infection, surgery should be postponed whenever possible. In these cases, alternate strategies should be employed when applicable (antibiotics, percutaneous drainage, endoscopic procedures, etc.). Implementation of new technologies or techniques should be avoided to reduce the operating time and the risk of postoperative complications [6]. For these reasons, all procedures should be performed by experienced surgeons with the minimum number of staff involved [16, 17].

\section{A) Anesthetic management}

All anesthetic equipment, appliances, and drugs must be specially assigned. The use of awake intubation techniques should be avoided. Fiberscope intubation should be avoided as well since it may provoke aerosolization. Fast induction of anesthesia with adequate muscle relaxation is recommended to prevent cough. The order of drug administration should be muscle relaxation drugs, intravenous general anesthetics, and opioids while trying to avoid mask pressurization ventilation before the patient loses consciousness.

During anesthesia maintenance, a small tidal volume of lung protective ventilation strategy should be adopted to reduce ventilator-related lung injury. A tidal volume of $4-8 \mathrm{~mL} / \mathrm{kg}$ ideal body weight, inspiratory plateau pressure less than $30 \mathrm{~cm} \mathrm{H} 2 \mathrm{O}$, PEEP level less than $8 \mathrm{~cm} \mathrm{H} 2 \mathrm{O}$, and recruitment maneuvers per $30 \mathrm{~min}$ are preferred. Ventilation parameters should be adjusted by blood gas analysis and PetCO2 during the operation [21].

\section{B) Laparoscopic vs. laparotomic approach}

SARS-CoV-2 is detected primarily from the human's upper and lower respiratory tract specimens. The virions are approximately 0.125 microns in size and can be transmitted as respiratory water droplets ( $>20$ microns) or aerosolized in smaller droplets ( $<10$ microns) in gas suspension. Viral RNAs can also be found in blood and stool specimens, but it is still not known if these specimens are infectious [22].
There are still controversies regarding the use of laparoscopic surgery in emergency settings when dealing with patients with COVID-19 [16, 19, 20]. The American College of Surgeons (ACS) has stated that there is insufficient data to recommend for/against an open versus laparoscopy approach. In laparoscopic surgery, an essential part of the technique is the establishment, maintenance, and evacuation of pneumoperitoneum (PNP), which puts the operating team under the risk of aerosol exposure. Ultrasonic scalpels or electrical equipment commonly used in laparoscopic surgery can easily produce large amounts of surgical smoke [23]. Previous studies have demonstrated that viruses such as hepatitis B, papillomavirus, and HIV can be detected in the surgical smoke during laparoscopic surgery [24-27]. Theoretically, the risk of SARS-CoV-2 containing aerosol transmission should not be an exception. At the same time, laparoscopic surgery has been extensively utilized for decades in these patients without any evidence of an increased risk of disease transmission to surgeons, anesthesiologists, or scrub nurses from surgical plumes or PNP. Moreover, other coronaviruses like SARS, MERS-CoV, and influenza virus have not been shown to cause transmission of disease through aerosolized laparoscopic CO2. Finally, a minimally invasive approach is much preferred in these settings since it can reduce exposure of surgeons to blood-borne pathogens, allowing for a self-contained operative field with very limited if any spillage of fluids and tissues [19-28]. Another option would be robotic surgery that allows for the OR staff and the surgeons to be remote from the patient and from each other [29].

It is now known that SARS-CoV-2 is also present in the stools of COVID-19 patients. Although the viral transmission during laparoscopic procedures has not yet been described and fecal-oral transmission has not been reported, specific attention must be paid to laparoscopic procedures needing bowel handling or a transperitoneal approach [30]. It has been demonstrated that after using electrical or ultrasonic equipment for $10 \mathrm{~min}$, the particle concentration of the smoke in laparoscopic surgery was significantly higher than that in traditional open surgery [26]. This could be due to the low gas mobility in the PNP, causing the aerosols formed during the operation to concentrate in the abdominal cavity. To date, no study has identified SARS-CoV-2 in surgical smoke, and even if once found, it is not known whether these viral particles will be contagious.

\section{C) Precautions during laparoscopic approach}

Despite the absence of definite data regarding the risk of SARS-CoV-2 transmission during laparoscopy, some precautions should be taken.

Prolonged Trendelenburg positioning should be avoided due to the deleterious effects on the cardiopulmonary 
function of COVID-19 patients. Efforts should be taken to reduce the amount and size of trocars as well as the incisions made to insert them. The intra-abdominal $\mathrm{CO}_{2}$ pressure should be kept lower than usual and possibly below $10 \mathrm{mmHg}$ and sudden release of trocar valves and non-airtight exchange of instruments must be avoided. The utilization of energy devices should be minimized and the use of a smoke evacuation/filtration system is encouraged. The Society of the American Gastrointestinal and Endoscopic Surgeon (SAGES) and the European Association for Endoscopic Surgery (EAES) advocate for the use of filters for the released $\mathrm{CO}_{2}$ during laparoscopy and robotic surgery [31]. Gas evacuation and filtration can be performed using an ultralow particulate air filter (ULPA) able to intercept particles as big as 0.1 microns in diameter (e.g., CONMED's AirSeal ${ }^{\circledR}$ system, Medtronic's RapidVac ${ }^{\text {TM }}$ or Stryker's PneumoClear ${ }^{\mathrm{TM}}$, J\&J's Megadyne ${ }^{\mathrm{TM}}$ ), while high-efficiency particulate air filters (HEPA) can stop particles as big as 0.3 microns in diameter. Additionally, before the final evacuation of the PNP or performing a mini-laparotomy, $\mathrm{CO} 2$ should be evacuated through an evacuation filter or connecting the trocar valve through a tube directly to a suction canister [31, 32].

Taking all the precautions, if not safer, laparoscopy can be considered as safe as laparotomy since, as previously described, it limits surgeons' exposure to the patient's blood and particle-containing aerosols. As compared to laparoscopy, containment of smoke is much more difficult during laparotomy increasing the risk of direct viral exposure of the surgical team. Moreover, laparotomy is followed by prolonged hospital stays and hospital bed utilization, an increased likelihood of ICU stay, and greater risk of post-operative complications (including undesirable pulmonary issues). Finally, the technical challenges associated with surgery performed via laparotomy in obese patients must be taken into consideration. Increased abdominal wall thickness can pose additional technical challenges during laparotomy limiting hand movements and visual scope, leading to an increase in operating time and potential risk of postoperative complications [28].

In conclusion, laparoscopy performed by an experienced surgical team, while taking all the previously described precautions, can be considered safe when dealing with COVID 19 patients. If clinically indicated, laparoscopic surgery optimizes patient's care and outcomes, while minimizing the contagious risk to the surgical team. The final recommendation is to evaluate the risk/benefit ratio concerning the use of a minimally invasive approach in COVID-19 patients. The "best approach" can be different between hospitals based on the availability of testing, PPE availability, or prevalence of the virus. This is particularly important in cases of morbidly obese patients that need emergency surgery since in this setting, the risks of a laparotomy far outweigh the risks of laparoscopy, even when taking into account practical concerns for the surgical team regarding potential exposure issues.

Awaiting further studies, we advise caution during laparoscopic procedures in morbidly obese patients with suspected or known COVID-19 and implementation of the preliminary guidelines suggested by Zheng et al. [23] as shown in Table 2.

Table 2 Precautions during laparoscopic surgery in patients with known or suspected COVID-19 (modified recommendations by Zheng et al.)

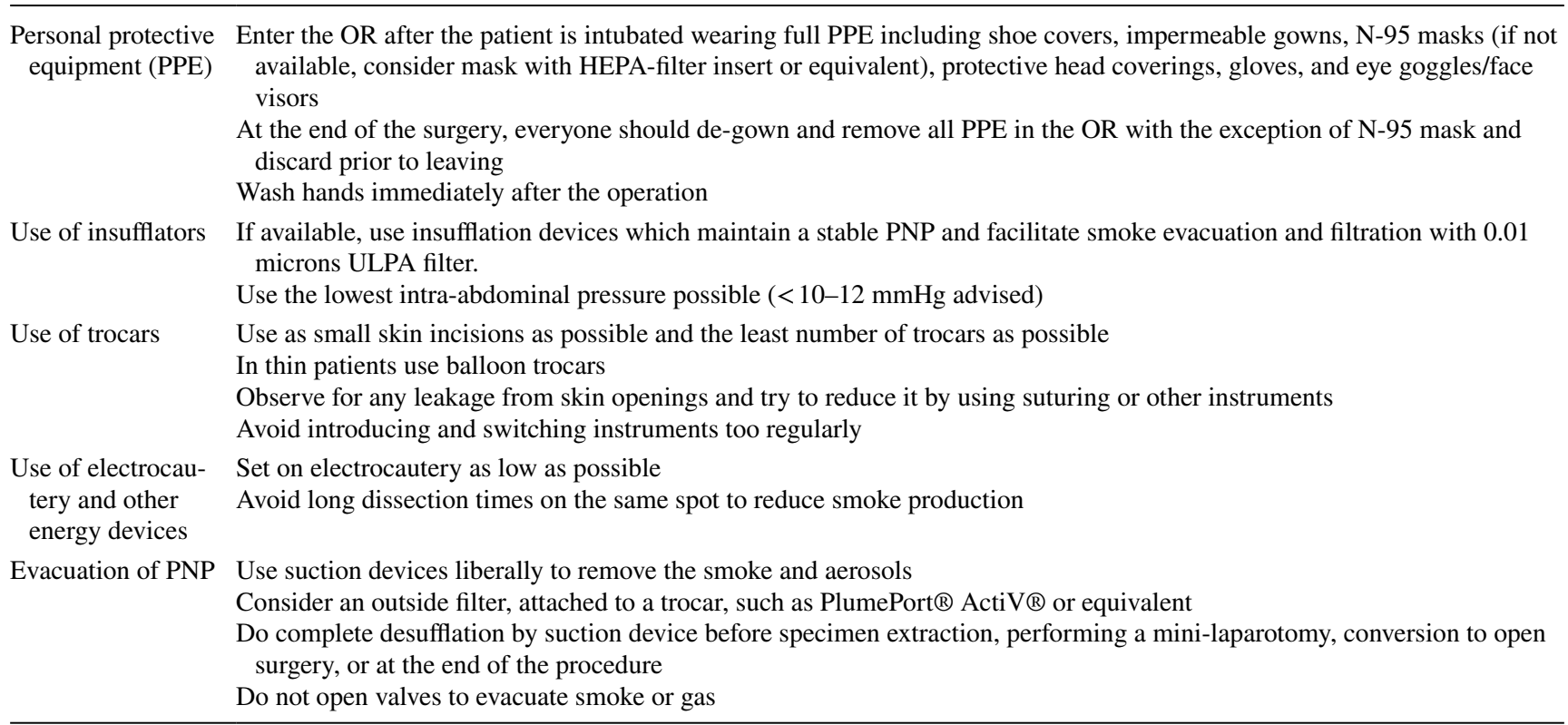




\section{How and when it is advisable to resume bariatric surgery in phase 2}

The WHO suggests that health systems should plan in advance how to recover after a pandemic but there are not precise guidelines on how exactly this should be done [33]. When hospitals finally begin to recover from the acute phase of the pandemic, the demand for elective care will increase, causing new pressure on the system. Surgeons must anticipate these scenarios to limit disruption. Therefore, planning the resumption of activities well in advance is crucial.

In a country like Italy where there are great differences in virus prevalence between regions, bariatric programs should not restart everywhere simultaneously. In fact, regions like Campania, Sicily, Calabria, and generally speaking, all the southern regions where the prevalence is less than $10 \%$, could restart soon after the end of the first phase of lockdown scheduled on the 4th of May, 2020. Regions as Lombardy, Emilia-Romagna, Piemonte and Veneto, where virus prevalence is much higher, should still wait. The Italian Prime Minister and each regional Governor will notify health care providers and the general population in detail when and which non-urgent outpatient activities and elective surgeries for benign disease can restart. Priority has to be given to protect patients and health care professionals from possible contagion. Recently, a decision-support tool for hospital leadership has been developed to understand not only the community exposure risk for healthcare workers, but also cross-infection between patients during this rapidly evolving pandemic, supporting universal masking PPE decisions. Since as many as $50 \%$ of all SARS-CoV-2 infections are asymptomatic, the prevalence of asymptomatic SARS$\mathrm{CoV}-2$ infections is roughly equivalent to the prevalence of known cases in a particular community assuming universal testing is available. With their tool, they estimate the risk that 1 out of every 1000 healthcare providers who see 20 patients per day without PPE would contract SARS-CoV-2, while with the use of PPE, this risk would drop to 3 out of every 10,000 . This information can be utilized throughout the epidemic to determine when de-escalation of these protocols should begin and stratify the risk of contagion between patients and health care providers and to modify the modality of outpatient visits within each hospital [34].

Bariatric surgery programs are generally based on recruitment and follow-up of patients through outpatient clinics and performing surgical procedures as in-patients. For both patients and health care providers, safety is the priority. Since the virus will circulate among human beings for years, in hospitals where infectious disease units operate, a different path has to be maintained for COVID-19 patients.

As a common rule, outpatient clinics should run again only when low virus prevalence is reached in that particular area or region. Clinics should possibly be located away from the hospitalization area. Pre-appointment phone screening should be implemented acquiring epidemiologic history. Suspected cases are canceled and reported to their general practitioners. Patients, whenever possible, should enter the hospital unaccompanied. Health care providers should utilize PPE, including surgical or N-95 masks, eye protection, and gloves. To allow social distancing, time allocated between appointments need to be longer, surgeons need to run outpatient on time, and patients should enter the hospital on time wearing masks and gloves. Meticulous cleaning of facilities and equipment should be maintained.

In particular, care must be taken to minimize in-person patient attendance and assess telemedicine with remote consultations. Contact patients by telephone to communicate results of laboratory, endoscopic, or radiological tests, whenever possible. If face-to-face consultation is essential, it is recommended to contact the patient the day before each appointment to screen them for fever or respiratory symptoms, to rule out any contact with a COVID-19 patient, and to make sure that they do not come from a high prevalence area.

As far as bariatric surgery is concerned, since important financial resources have been consumed during the acute phase of the pandemic and the health system will have to deal with a big backlog of cases, a triage is crucial to reassess patients and considering referring them to centers with greater capacity, if resources become scarce. Once the activity is resumed, it is mandatory to regularly and realistically assess the hospital to ensure system's capacity and to expand progressively surgical services, if indicated. In this setting, developing an approach to prioritization of cases that have waited variable amounts of time will be crucial.

Before admission, patients should undergo an interview to rule out the possible SARS-CoV-2 infection. They should be screened for fever or respiratory symptoms, to be asked about any contact with a COVID-19 patient, and be assessed whether they come from a high prevalence area. All patients should undergo a preoperative nasopharyngeal sample for SARS-CoV-2 and/or a rapid test (IgG/IgM dosage) on admission.

The informed consent acquisition process needs to be adjusted due to the COVID-19 pandemic. Some questions still have to be answered: symptomatic or previously symptomatic COVID-19 status impact on postoperative complications is still unknown; SARS-CoV-2 acquisition risk for a patient coming to the hospital is uncertain; also the risks inherent in delaying an otherwise clinically indicated procedures are still not known. While the increased risk of cardiorespiratory and thrombotic complications in symptomatic COVID-19-positive patients is confirmed, we still do not know if asymptomatic or previously symptomatic individuals undergoing an operation or general anesthesia run some risk $[35,36]$. In addition, hospitals have implemented 
visitors' restriction policies that limit the possibility for patients to communicate with friends and family. Finally, because of the shortage of facilities associated with the pandemic, it may impact some patients' hospital stay. All these dimensions need to be discussed with patients prior to admission, or prior to scheduling the surgery, to let the single individuals decide to proceed with or postpone the surgery.

Clinical judgments by the multidisciplinary team will guide the prioritization of patients on the waiting list [7]. In low virus prevalence regions, SICOB guidelines on bariatric surgery indications can be followed. However, in high-virusprevalence regions, priority will be given to revisional surgery, patients with new-onset or worsening of comorbidities, and patients with significant weight gain over the last few months.

To minimize cross-infection, transfers within the hospital have to follow COVID-19-free paths, and ORs have to be no-COVID [16]. Hospital staff, at all times, and patients, whenever possible should wear a mask. Surgery should be scheduled the day after admission with a laparoscopic approach using the precautions already discussed earlier. ERAS protocols should be implemented to speed up the recovery process, minimize the postoperative hospital stay, and allow the discharge of patients not later than the second postoperative day. Follow-up will be a mix of face-to-face visits and online consultation to allow social distancing within hospitals and minimize the chance of contagion for patients [37, 38].

\section{The second wave: possible scenarios}

Easing measures aimed to limit social contacts, resumption of economic activities, progressive and continuous virus spread worldwide with possible importation of new cases from abroad and residual infected foci within Italy increase the likelihood of a second wave of COVID-19 in autumn 2020. However, the three T-approach (Test, Track, Trace) should be able to limit its impact [39]. The most likely scenario in Italy will be of several foci scattered along the peninsula that will be put under temporary lockdown, similar to the red zone instituted in the Milan area at the beginning of March. In this case, there will be no or very limited impact on the NHS nationwide. If there is wider diffusion of the virus, entire regions or the whole nation will be put on lockdown again. For this situation, each region or the entire nation will be back on phase 1, halting non-urgent/non-oncologic surgery and outpatients. However, the NHS should be prepared for this eventuality and COVID-19 hospitals or mixed hospitals with completely separate paths for COVID19-non-COVID-19 patients will be established. In such cases, the central government will coordinate procedures.

\section{Educational considerations during the COVID-19 pandemic}

Reduction of residents on duty within each hospital coupled to cancellation of conferences and seminars to obey social distancing, together with the delay of elective cases and reduction of emergency surgeries has already led to a significant alteration of the training processes. Several strategies can be implemented to overcome the above-mentioned restrictions. Dual coding theory by Allan Paivio is the base of operative video-based education that is increasingly utilized worldwide [40]. Formal teaching can be maintained using video-based conferencing platforms that allow mentors and trainees to meet regularly. These platforms offer lecturers the ability to share presentations in real time as well as to view the session outside the scheduled time. Trainees are also encouraged to implement self-directed learning and to search the internet for additional learning material through surgical societies, curated content on podcasts, and social media [41]. Virtual simulation can be done individually, even after hours, and performance can be reviewed remotely or recorded and uploaded.

\section{Conclusion}

COVID-19 pandemic has found the global surgical community unprepared to provide an appropriate and prompt response, hence, heavily affecting surgical activities. The authors have tried to provide recommendations regarding the triage of surgical procedures in morbidly obese patients during the COVID-19 pandemic that may require emergency surgery and to delineate a strategy to safely resume bariatric surgery programs after the acute phase of the pandemic. Models developed during the COVID-19 crisis should be integrated into hospital practices for future use in similar scenarios. Surgeons have been presented with a golden opportunity to embrace systemic change and to drive their professional future.

Author contributions All authors contributed to the study conception and design. The first draft of the manuscript was written by GN and IK. All authors read, commented, and approved the final manuscript.

Data availability Not applicable because of the type of this article.

\section{Compliance with ethical standards}

Conflict of interest The authors declare that they have no conflict of interest.

Research involving human participants and/or animals This article did not contain any studies with human participants or animals performed by any of the authors. 
Ethical approval Ethical committee approval was waived because of the type of this article.

Informed consent This study did not involve human participants so informed consent were not needed.

\section{References}

1. Weiss SR, Leibowitz JL (2011) Coronavirus pathogenesis. Adv Virus Res 81:85-164

2. Tan W, Zhao X, Ma X et al (2020) Notes from the field: a novel coronavirus genome identified in a cluster of pneumonia casesWuhan, China 2019-2020. China CDC Wkly 2:61-62

3. Wang D, Hu B, Hu C et al (2020) Clinical characteristics of 138 hospitalized patients with 2019 novel coronavirus-infected pneumonia in Wuhan, China. JAMA 323:1061-1069

4. World Health Organization (WHO) (2020) Coronavirus disease (COVID-19) situation dashboard. https://covid19.who.int/region/ euro/country/it. Accessed 3 May 2020

5. Remuzzi A, Remuzzi G (2020) COVID-19 and Italy: what next? Lancet 395:1225-1228

6. Ficarra V, Novara G, Abrate A et al (2020) Urology practice during COVID-19 pandemic. Minerva Urol Nefrol. https://doi. org/10.23736/S0393-2249.20.03846-1

7. Diaz A, Sarac BA, Schoenbrunner AR et al (2020) Elective surgery in the time of COVID-19. Am J Surg. https://doi. org/10.1016/j.amjsurg.2020.04.014

8. Iacobucci G (2020) Covid-19: all non-urgent elective surgery is suspended for at least three months in England. BMJ. https://doi. org/10.1136/bmj.m1106

9. Grasselli G, Zangrillo A, Zanella A et al (2020) Baseline characteristics and outcomes of 1591 patients infected with SARSCoV-2 admitted to ICUs of the Lombardy Region, Italy. JAMA 323:1574-1581

10. Kass DA, Duggal P, Cingolani O (2020) Obesity could shift severe COVID-19 disease to younger ages. Lancet. https://doi. org/10.1016/S0140-6736(20)31024-2

11. Dietz W, Santos-Burgoa C (2020) Obesity and its implications for COVID-19 mortality. Obesity. https://doi.org/10.1002/oby.22818

12. Sattar N, McInnes IB, McMurray JJV (2020) Obesity a risk for severe COVID-19 infection: multiple potential mechanisms. Circulation. https://doi.org/10.1161/CIRCULATIONAHA.120.04765 9

13. Lighter J, Phillips M, Hochman S et al (2020) Obesity in patients younger than 60 years is a risk factor for Covid-19 hospital admission. Clin Infect Dis. https://doi.org/10.1093/cid/ciaa415

14. Simonnet A, Chetboun M, Poissy J et al (2020) Intensive Care COVID-19 and Obesity study group. High prevalence of obesity in severe acute respiratory syndrome coronavirus-2 (SARS-Co-2) requiring invasive mechanical ventilation. Obesity. https://doi. org/10.1002/oby.22831

15. American College of Surgeons (2020) COVID-19 guidelines for triage of metabolic and bariatric surgery patients. https://www. facs.org/covid-19/clinical-guidance/elective-case/metabolic-baria tric. Accessed 24 Mar 2020

16. Coimbra R, Edwards S, Kurihara H et al (2020) European Society of Trauma and Emergency Surgery (ESTES) recommendations for trauma and emergency surgery preparation during times of COVID-19 infection. Eur J Trauma Emerg Surg. https://doi. org/10.1007/s00068-020-01364-7

17. Coccolini F, Perrone G, Chiarugi M et al (2020) Surgery in COVID-19 patients: operational directives. World J Emerg Surg $15: 25$
18. Ti LK, Ang LS, Foong TW et al (2020) What we do when a COVID-19 patient needs an operation: operating room preparation and guidance. Can J Anaesth. https://doi.org/10.1007/s1263 0-020-01617-4

19. Mallick R, Odejinmi F, Clark TJ (2020) COVID 19 pandemic and gynecological laparoscopic surgery: knowns and unknowns. Facts Views Vis ObGyn 12:3-7

20. Vigneswaran Y, Prachand VN, Posner MC et al (2020) What is the appropriate use of laparoscopy over open procedures in the current COVID-19 climate? J Gastrointest Surg. https://doi. org/10.1007/s11605-020-04592-9

21. Wen X, Li Y (2020) Anesthesia procedure of emergency operation for patients with suspected or confirmed COVID 19. Surg Infect 21:1

22. Yeo C, Kaushal S, Yeo D (2020) Enteric involvement of coronaviruses: is faecal-oral transmission of SARS-CoV-2 possible? Lancet Gastroenterol Hepatol 5:335-337

23. Zheng MH, Boni L, Fingerhut A (2020) Minimally invasive surgery and the novel coronavirus outbreak: lessons learned in China and Italy. Ann Surg. https://doi.org/10.1097/SLA.00000 00000003924

24. Alp E, Bij1 D, Bleichrodt RP (2006) Surgical smoke and infection control. J Hosp Infect 62:1-5

25. Kwak HD, Kim SH, Seo YS (2016) Detecting hepatitis B virus in surgical smoke emitted during laparoscopic surgery. Occup Environ Med 73:857-863

26. Hensman C (1998) Chemical composition of smoke produced by high-frequency electrosurgery in a closed gaseous environment. Surg Endosc 12:1017-1019

27. Gloster HM, Roenigk RK (1995) Risk of acquiring human papillomavirus from the plume produced by the carbon dioxide laser in the treatment of warts. J Am Acad Dermatol 32:436-441

28. Ciarrocchi A, Amicucci G (2014) Laparoscopic versus open appendectomy in obese patients: a meta-analysis of prospective and retrospective studies. J Minim Access Surg 10:4-9

29. Kimmig R, Verheijen RHM, Rudnicki M (2020) Robot assisted surgery during the COVID-19 pandemic, especially for gynecological cancer: a statement of the Society of European Robotic Gynaecological Surgery (SERGS). J Gynecol Oncol 31:e59

30. Li CI, Pai JY, Chen CH (2020) Characterization of smoke generated during the use of surgical knife in laparotomy surgeries. $\mathbf{J}$ Air Waste Manag Assoc 70:324-332

31. EAES-SAGES joint recommendations (2010) Resources for smoke \& gas evacuation during open, laparoscopic, and endoscopic procedures. https://eaes.eu/resources-on-smoke-gasevacuation-during-open-laparoscopic-and-endoscopic-proce dures/. Accessed 26 Mar 2020

32. Mintz Y, Arezzo A, Boni L et al (2020) A low cost, safe and effective method for smoke evacuation in laparoscopic surgery for suspected coronavirus patients. Ann Surg. https://doi. org/10.1097/SLA.0000000000003965

33. World Health Organization (2009) Pandemic influenza preparedness and response. A WHO guidance document. https:// apps.who.int/iris/bitstream/handle/10665/44123/9789241547 680_eng.pdf;jsessionid=E13C4C284916D0689FD66BBA3 CBB04E4? sequence $=1$. Accessed 5 May 2020

34. Graham LA, Maldonado JA, Tompkins LS, et al (2020) Asymptomatic SARS-CoV-2 Transmission from Community Contacts in Healthcare Workers. Ann Surg. https://journals.lww.com/ annalsofsurgery/Documents/AsymptomaticSARSCoV2Trans mission.pdf. Accessed 10 May 2020

35. Guo T, Fan Y, Chen M (2020) Cardiovascular implications of fatal outcomes of patients with coronavirus disease 2019 (COVID-19). JAMA Cardiol. https://doi.org/10.1001/jamac $\operatorname{ardio.2020.1017}$ 
36. Zhou F, Du R, Fan G et al (2020) Clinical course and risk factors for mortality of adult inpatients with COVID-19 in Wuhan, China: a retrospective cohort study. Lancet 395:1054-1062

37. Trotta M, Ferrari C, D'Alessandro G et al (2019) Enhanced recovery after bariatric surgery (ERABS) in a high-volume bariatric center. Surg Obes Relat Dis 15:1785-1792

38. Dang JT, Szeto VG, Elnahas A et al (2020) Canadian consensus statement: enhanced recovery after surgery in bariatric surgery. Surg Endosc 34:1366-1375

39. Leung K, Wu JT, Leung GM (2020) First-wave COVID-19 transmissibility and severity in China outside Hubei after control measures, and second-wave scenario planning: a modelling impact assessment. Lancet 395:1382-1393
40. Ahmet A, Gamze K, Rustem M et al (2018) Is video-based education an effective method in surgical education? A systematic review. J Surg Educ 75:1150-1158

41. Artino AR Jr, Brydges R, Gruppen LD (2015) Self-regulated learning in healthcare profession education: theoretical perspectives and research methods. In: Cleland J, Durning SJ (eds) Researching medical education. West Sussex, UK, pp 155-166

Publisher's Note Springer Nature remains neutral with regard to jurisdictional claims in published maps and institutional affiliations. 